\title{
Characterization of $q$-Cesàro convergence for double sequences
}

\author{
Emre Taş and Cihan Orhan
}

\begin{abstract}
In the present paper we examine the Buck-Pollard property of 4dimensional $q$-Cesàro matrices. Indeed we discuss some questions related to the $q$-Cesàro summability of subsequences of a given double sequence. The main result states that " a bounded double sequence is $q$-Cesàro summable to $L$ if and only if almost all of its subsequences are $q$-Cesàro summable to $2^{1-q} L "$.
\end{abstract}

Mathematics Subject Classification (2010): 40B05, 40C05, 28A12.

Keywords: Double sequences, Pringsheim convergence, the Buck-Pollard property, $q$-Cesàro matrices.

\section{Introduction}

Buck and Pollard [2] proved that a bounded sequence $\left(s_{n}\right)$ is $(C, 1)$ summable if and only if almost all of its subsequences is $(C, 1)$ summable. Since this idea has been introduced by Buck and Pollard, the property is to be called "Buck-Pollard property". The Buck-Pollard property is related to the convergence or summability of subsequences of a given sequence. Taking into consideration $q$-Cesàro matrix instead of $(C, 1)$ matrix, similar results have been investigated in [7]. Recently the Buck-Pollard property for $(C, 1,1)$ summability method has been examined and also provided a new characterization of $(C, 1,1)$ summability for double sequences with respect to its subsequences [10].

In the present paper we consider similar problems for four dimensional q-Cesàro matrix on double sequences. We first introduce the notions of our interest related to double sequences.

A double sequence $s=\left(s_{j k}\right)$ is said to be Pringsheim convergent (i.e., it is convergent in Pringsheim's sense) to $L$ if for every $\varepsilon>0$ there exists an $N \in \mathbb{N}$ such that $\left|s_{j k}-L\right|<\varepsilon$ whenever $j, k \geq N([9])$. In this case $L$ is called the Pringsheim limit of $s$ and the space of such sequences is denoted by $c^{(2)}$. A double sequence $s$ is 
bounded if there exists a positive number $H$ such that $\left|s_{j k}\right|<H$ for all $j$ and $k$, i.e.,

$$
\|s\|_{(\infty, 2)}=\sup _{j, k}\left|s_{j k}\right|<\infty .
$$

We will denote the set of all bounded double sequences by $l_{\infty}^{(2)}$. Note that in contrast to the case for single sequences, a convergent double sequence need not to be bounded.

Throughout the paper when there is no confusion, "convergence" means the Pringsheim convergence.

Four dimensional $q$-Cesàro matrix $\left(C_{q}, 1,1\right)=\left(c_{j k}^{n m}\right)$ is defined by

$$
c_{j k}^{n m}=\left\{\begin{array}{ccc}
\frac{1}{n^{q} m^{q}} & , & 1 \leq j \leq n \text { and } 1 \leq k \leq m \\
0 & , & \text { otherwise }
\end{array}\right.
$$

where $0<q<\infty$. Observe that the case $q=1$ reduces to $(C, 1,1)$, 4-dimensional Cesàro matrix. Also if $q \neq 1, q$-Cesàro matrices $\left(C_{q}, 1,1\right)$ cannot be $R H$ regular, i.e., it cannot sum every bounded convergent sequence to the same limit.

There exist several versions of the concept of subsequences for double sequences ([3], [8], [12]). We adopt Definition 2 of [3] on subsequences of double sequences throughout the paper.

Let $X$ denote the set of all double sequences of 0's and 1's, that is

$$
X=\left\{x=\left(x_{j k}\right): x_{j k} \in\{0,1\} \text { for each } j, k \in \mathbb{N}\right\} .
$$

Let $\Re$ be the smallest $\sigma$-algebra of subsets of the set $X$ which contains all sets of the form

$$
\left\{x=\left(x_{j k}\right) \in X: x_{j_{1} k_{1}}=a_{1}, \ldots, x_{j_{n} k_{n}}=a_{n}\right\}
$$

where each $a_{i} \in\{0,1\}$ and the pairs $\left\{\left(j_{i} k_{i}\right)\right\}_{i=1}^{n}$ are pairwise distinct.

There exists a unique probability measure $P$ on the set $\Re$, such that

$$
P\left(\left\{x=\left(x_{j k}\right) \in X: x_{j_{1} k_{1}}=a_{1}, \ldots, x_{j_{n} k_{n}}=a_{n}\right\}\right)=\frac{1}{2^{n}}
$$

for all choices of $n$ and all pairwise disjoint pairs $\left\{\left(j_{i} k_{i}\right)\right\}_{i=1}^{n}$, and all choices of $a_{1}, \ldots, a_{n}$ (see, [3]).

Let $s=\left(s_{j k}\right)$ be a double sequence and $x=\left(x_{j k}\right) \in X$. Following [3] we define a subsequence of the sequence $s$ by

$$
s_{j k}(x)=\left\{\begin{array}{cll}
s_{j k} & , & \text { if } x_{j k}=1 \\
* & , & \text { if } x_{j k}=0
\end{array} .\right.
$$

Mapping $x \rightarrow s(x)$ is a bijection from the set $X$ to the set of all the subsequences of the sequence $s=\left(s_{j k}\right)$ [3].

An element $x$ of $X$ is said to be normal ([3]) if for each $\varepsilon>0$ there is a natural number $N_{\varepsilon}$ such that for $n, m \geq N_{\varepsilon}$ we have $\left|\frac{1}{n m} \sum_{\substack{j \leq n \\ k \leq m}} x_{j k}-\frac{1}{2}\right|<\varepsilon$. Let $\eta$ denote the set of all elements $x$ in $X$ that are normal. This implies that normal elements are $(C, 1,1)$-summable to $\frac{1}{2}$. It is also known $([3])$ that $P(\eta)=1$. 


\section{Subsequence Characterization of $q$-Cesàro Summability}

In this section we characterize $\left(C_{q}, 1,1\right)$ summability of a double sequence. In particular we study conditions under which $\left(C_{q}, 1,1\right)$ summability of a double sequence carry over to that of its subsequences, and conversely, whether these properties for suitable subsequences imply them for the sequence itself. We begin with the following theorem which is analog to that of Buck and Pollard [2] for single sequences.

Theorem 2.1. If almost all subsequences of $s=\left(s_{j k}\right)$ are $\left(C_{q}, 1,1\right)$-summable to a value $L$ then the sequence $s=\left(s_{j k}\right)$ is $\left(C_{q}, 1,1\right)$-summable to $2^{1-q} L$.

Proof. If almost all subsequences of $\left(s_{j k}\right)$ are $\left(C_{q}, 1,1\right)$-summable to a value $L$ then the set $G=\left\{x \in X: s(x)\right.$ is $\left(C_{q}, 1,1\right)$-summable to $\left.L\right\}$ has probability measure 1 . We use the technique given in [3]. Now given a sequence $x=\left(x_{j k}\right) \in X$ we define a sequence $\bar{x}=\left(\bar{x}_{j k}\right)$ by

$$
\bar{x}_{j k}=\left\{\begin{array}{ll}
0, & \text { if } x_{j k}=1 \\
1 & , \text { if } x_{j k}=0
\end{array} .\right.
$$

Let $Y=G \cap \eta$ and $\bar{Y}=\left\{\left(\bar{x}_{j k}\right): x_{j k} \in Y\right\}$. Therefore we have $\bar{Y}=\bar{G} \cap \eta$ where $\bar{G}$ is defined in the obvious way. Since the mapping $\left(x_{j k}\right) \rightarrow\left(\bar{x}_{j k}\right)$ preserves the measure $P$, we get $P(\bar{Y})=1$ and hence $P(Y \cap \bar{Y})=1$. So $Y \cap \bar{Y}$ is a non-empty set. If $x=\left(x_{j k}\right) \in Y \cap \bar{Y}$, then we have $x \in G, x \in \eta$ and $\bar{x} \in G, \bar{x} \in \eta$. Hence we obtain

$$
s(x) \rightarrow L\left(C_{q}, 1,1\right)
$$

and

$$
s(\bar{x}) \rightarrow L\left(C_{q}, 1,1\right)
$$

with $x, \bar{x} \in \eta$. That is

$$
\lim _{n, m \rightarrow \infty} \frac{\sum_{j, k=1,1}^{n, m} s_{j k} x_{j k}}{\left(\sum_{j, k=1,1}^{n, m} x_{j k}\right)^{q}}=L \text { and } \lim _{n, m \rightarrow \infty} \frac{\sum_{j, k=1,1}^{n, m} s_{j k} \bar{x}_{j k}}{\left(\sum_{j, k=1,1}^{n, m} \bar{x}_{j k}\right)^{q}}=L .
$$

Also since $x, \bar{x} \in \eta$, we have

$$
\lim _{n, m \rightarrow \infty} \frac{1}{n m} \sum_{j, k=1,1}^{n, m} x_{j k}=\frac{1}{2} \text { and } \lim _{n, m \rightarrow \infty} \frac{1}{n m} \sum_{j, k=1,1}^{n, m} \bar{x}_{j k}=\frac{1}{2} .
$$

On the other hand, the $\left(C_{q}, 1,1\right)$-summability of the sequence $\left(s_{j k}\right)$ is equivalent to the existence of the limit of the following expression

$$
\frac{\sum_{j, k=1,1}^{n, m} s_{j k}}{n^{q} m^{q}}=\frac{\left(\sum_{j, k=1,1}^{n, m} x_{j k}\right)^{q}}{n^{q} m^{q}} \frac{\sum_{j, k=1,1}^{n, m} s_{j k} x_{j k}}{\left(\sum_{j, k=1,1}^{n, m} x_{j k}\right)^{q}}+\frac{\left(\sum_{j, k=1,1}^{n, m} \bar{x}_{j k}\right)^{q}}{n^{q} m^{q}} \frac{\sum_{j, k=1,1}^{n, m} s_{j k} \bar{x}_{j k}}{\left(\sum_{j, k=1,1}^{n, m} \bar{x}_{j k}\right)^{q}} .
$$


So we get that

$$
\lim _{n, m \rightarrow \infty} \frac{\sum_{j, k=1,1}^{n, m} s_{j k}}{n^{q} m^{q}}=\frac{L}{2^{q}}+\frac{L}{2^{q}}=2^{1-q} L
$$

which implies that the sequence $\left(s_{j k}\right)$ is $\left(C_{q}, 1,1\right)$-summable to $2^{1-q} L$.

In order to get the converse of Theorem 2.1, we need the following two lemmas presented in [10]. The first lemma is an analog of the Khintchine inequality for double sequences.

Lemma 2.2. Let

$$
t_{n m}(x)=\sum_{j, k=1,1}^{n, m} s_{j k} r_{j k}(x), \quad B_{n m}=\sum_{j, k=1,1}^{n, m} s_{j k}^{2} .
$$

Then the following inequality

$$
E\left(\left(t_{n m}\right)^{2 r}\right) \leq \frac{(2 r) !}{2^{r} r !}\left(B_{n m}\right)^{r}
$$

is fulfilled, where $r$ is a positive integer.

The next result is an analog of the Marcinkiewicz-Zygmund inequality for double sequences.

Lemma 2.3. Let

$$
t_{n m}(x)=\sum_{j, k=1,1}^{n, m} s_{j k} r_{j k}(x), \quad B_{n m}=\sum_{j, k=1,1}^{n, m} s_{j k}^{2}
$$

and $t_{n m}^{*}(x)=\max _{(j, k) \in K_{n m}}\left|t_{j k}\right|$, where $K_{n m}:=\{(j, k): 1 \leq j \leq n, 1 \leq k \leq m\}$.

Then for $a>0$ the following inequality

$$
E\left(e^{a t_{n m}^{*}(x)}\right) \leq 32 e^{a^{2} \frac{B_{n m}}{2}}
$$

holds.

Now we are ready to provide the converse of Theorem 2.1.

Theorem 2.4. If the sequence $\left(s_{j k}\right)$ is $\left(C_{q}, 1,1\right)$-summable to a value $L$ and

$$
\sum_{j, k=1,1}^{n, m} s_{j k}^{2}=o\left(\frac{n^{2 q} m^{2 q}}{\log \log n^{q} m^{q}}\right)
$$

then almost all subsequences of $\left(s_{j k}\right)$ are $\left(C_{q}, 1,1\right)$-summable to $2^{q-1} L$.

Proof. The $\left(C_{q}, 1,1\right)$-summability of almost all subsequences of $\left(s_{j k}\right)$ is equivalent to the convergence of the following expression

$$
\frac{\sum_{j, k=1,1}^{n, m} s_{j k} x_{j k}}{\left(\sum_{j, k=1,1}^{n, m} x_{j k}\right)^{q}} \text { for almost all } x \text {. }
$$


We can rewrite the above expression as follows for almost all $x$

$$
\frac{\sum_{j, k=1,1}^{n, m} s_{j k}\left(\frac{1+r_{j k}(x)}{2}\right)}{\left\{\sum_{j, k=1,1}^{n, m}\left(\frac{1+r_{j k}(x)}{2}\right)\right\}^{q}}=\frac{\frac{1}{2 n^{q} m^{q}} \sum_{j, k=1,1}^{n, m} s_{j k}+\frac{1}{2 n^{q} m^{q}} \sum_{j, k=1,1}^{n, m} s_{j k} r_{j k}(x)}{\frac{1}{n^{q} m^{q}}\left\{\sum_{j, k=1,1}^{n, m}\left(\frac{1+r_{j k}(x)}{2}\right)\right\}^{q}}
$$

where $r_{j k}(x)=2 x_{j k}-1$. Recall that the functions $r_{j k}$ are the Rademacher functions (see [3]). Since $P(\eta)=1$, observe that the denumerator of $(2.1)$ converges to $\frac{1}{2^{q}}$ for almost all $x$. To complete the proof, it suffices to establish that

$$
\frac{1}{n^{q} m^{q}} \sum_{j, k=1,1}^{n, m} s_{j k} r_{j k}(x) \rightarrow 0,(\text { as } n, m \rightarrow \infty) \text { for almost all } x .
$$

Let $\varepsilon>0$ and define

$$
M_{j k}:=\left\{x \text { : there is }(n, m) \text { with } 2^{j-1}<n \leq 2^{j}, 2^{k-1}<m \leq 2^{k} \text { such that }\left|t_{n m}(x)\right| \geqslant n^{q} m^{q} \varepsilon\right\}
$$

and let

$$
G_{j k}=\left\{x: t_{2^{j}, 2^{k}}^{*}(x)>2^{q(j-1)} 2^{q(k-1)} \varepsilon\right\} .
$$

Notice that $M_{j k} \subset G_{j k}$. The proof will be completed if we prove that for every $\varepsilon>0$,

$$
\sum_{j, k=1,1}^{\infty, \infty} P\left(G_{j k}\right)<\infty
$$

Now using Lemma 2.3 we have

$$
P\left(G_{j k}\right) e^{a 2^{q(j-1)} 2^{q(k-1)} \varepsilon} \leq \int_{X} e^{a t_{2^{j}, 2^{k}}^{*}(x)} d P(x)=E\left(e^{a t_{2^{j}, 2^{k}}^{*}(x)}\right) \leq 32 e^{a^{2} \frac{B_{2^{j} 2^{k}}}{2}} .
$$

Hence

$$
P\left(G_{j k}\right) \leq 32 e^{\frac{a^{2} B_{2 j} k}{2}-a 2^{q(j-1)} 2^{q(k-1)} \varepsilon} .
$$

Taking $a=\frac{2^{q(j-1)} 2^{q(k-1)} \varepsilon}{B_{2^{j} 2^{k}}}$, we have

$$
\begin{aligned}
P\left(G_{j k}\right) & \leq 32 e^{-\frac{\varepsilon^{2} 2^{2 q(j-1)} 2^{2 q(k-1)}}{2 B_{2^{j} 2^{k}}}} \\
& =32 e^{-\frac{\varepsilon^{2}\left(2^{j}\right)^{2 q}\left(2^{k}\right)^{2 q}}{2.16^{q} B_{2^{j} 2^{k}}}} .
\end{aligned}
$$

On the other hand it follows from the hypothesis that

$$
\frac{B_{2^{j} 2^{k}}}{\left(2^{j}\right)^{2 q}\left(2^{k}\right)^{2 q}}=o\left(\frac{1}{\log \log 2^{j q} 2^{k q}}\right)
$$

which yields

$$
\frac{B_{2^{j} 2^{k}}}{\left(2^{j}\right)^{2 q}\left(2^{k}\right)^{2 q}} \leq \frac{\varepsilon^{2}}{2.16^{q} \log \log 2^{j q} 2^{k q}}
$$


Then (2.2) yields that

$$
\begin{aligned}
P\left(G_{j k}\right) & \leq 32 e^{-\frac{\varepsilon^{2}}{2.16^{q}} \frac{6.16^{q} \log \log 2^{j q} 2^{k q}}{\varepsilon^{2}}} \\
& =32 e^{-3 \log \log 2^{j q} 2^{k q}} \\
& =\frac{32}{\left[(j+k) \log 2^{q}\right]^{3}}
\end{aligned}
$$

Since $\sum_{j, k=1,1}^{\infty, \infty} \frac{1}{\left[(j+k) \log 2^{q}\right]^{3}}<\infty($ see $[1])$,

$$
\sum_{j, k=1,1}^{\infty, \infty} P\left(G_{j k}\right) \leq 32 \sum_{j, k=1,1}^{\infty, \infty} \frac{1}{\left[(j+k) \log 2^{q}\right]^{3}}<\infty .
$$

Hence we obtain $\lim _{j, k \rightarrow \infty} P\left(G_{j k}\right)=0$ and also $\lim _{j, k \rightarrow \infty} P\left(M_{j k}\right)=0$. This completes the proof.

A criterion for $\left(C_{q}, 1,1\right)$ summability of bounded double sequences is provided in the next corollary.

Corollary 2.5. A bounded double sequence $\left(s_{j k}\right)$ is $\left(C_{q}, 1,1\right)$-summable if and only if the almost all subsequences are $\left(C_{q}, 1,1\right)$-summable.

Theorem 2.6. If

$$
\lim _{n, m \rightarrow \infty} \frac{1}{n^{q} m^{q}} \sum_{j, k=1,1}^{n, m} s_{j k} r_{j k}(x)=0, \text { for almost all } x
$$

then we have

$$
\lim _{n, m \rightarrow \infty} \frac{1}{n^{2 q} m^{2 q}} \sum_{j, k=1,1}^{n, m} s_{j k}^{2}=0 .
$$

Proof. Let $N[u, z]=\{(j, k): u \leq j \leq n$ or $z \leq k \leq m\}$ and

$$
T_{u, z, n, m}(x)=\sum_{(j, k) \in N[u, z]} s_{j k} r_{j k}(x) .
$$

Hence

$$
T_{u, z, n, m}^{2}(x)=\sum_{(j, k) \in N[u, z]} s_{j k}^{2}+2 \sum_{\substack{\left(j_{1}, k_{1}\right),\left(j_{2}, k_{2}\right) \in N[u, z] \\ j_{1} \neq j_{2} \text { or } k_{1} \neq k_{2}}} s_{j_{1} k_{1}} s_{j_{2} k_{2}} r_{j_{1} k_{1}}(x) r_{j_{2} k_{2}}(x) .
$$

Because of the Egoroff theorem there exists a set $D \subset X$ with positive measure such that the limit in (2.3) exists uniformly on $D$. Therefore,

$$
\int_{D} T_{u, z, n, m}^{2}(x) d P(x)=P(D) \sum_{(j, k) \in N[u, z]} s_{j k}^{2}+K
$$


where

$$
K=2 \sum_{\substack{\left.j_{1}, k_{1}\right),\left(j_{2}, k_{2}\right) \in N[u, z] \\ j_{1} \neq j_{2} \text { or } k_{1} \neq k_{2}}} s_{j_{1} k_{1}} s_{j_{2} k_{2}} \int_{D} r_{j_{1} k_{1}}(x) r_{j_{2} k_{2}}(x) d P(x) .
$$

By the Hölder inequality we have

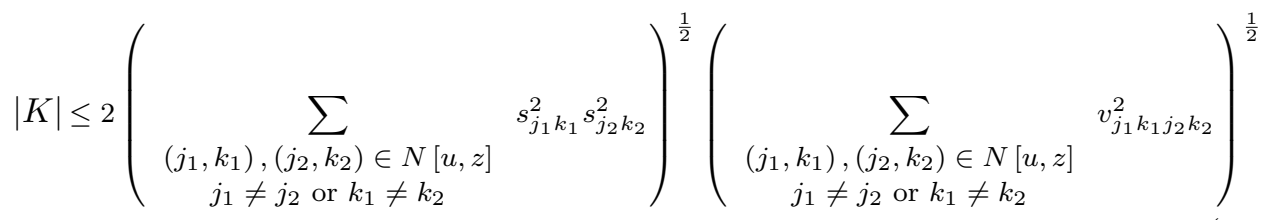

where $v_{j_{1} k_{1} j_{2} k_{2}}=\int_{D} r_{j_{1} k_{1}}(x) r_{j_{2} k_{2}}(x) d P(x)$. We know that the functions $r_{j_{1} k_{1}}(x)$ and $r_{j_{2} k_{2}}(x)$ are orthogonal on $X$ (see [3]). So by the Bessel inequality [13] for double sequences we get

$$
\sum_{\substack{1 \leq j_{1}<j_{2} \leq \infty \\ 1 \leq k_{1}<k_{2} \leq \infty}} v_{j_{1} k_{1} j_{2} k_{2}}^{2} \leq \int_{X}\left(\chi_{D}(x)\right)^{2} d P(x)=P(D) .
$$

For sufficiently large $u$ and $z$, we have

$$
\left(\sum_{\substack{\left.j_{1}, k_{1}\right),\left(j_{2}, k_{2}\right) \in N[u, z] \\ j_{1} \neq j_{2} \text { or } k_{1} \neq k_{2}}} v_{j_{1} k_{1} j_{2} k_{2}}^{2} \leq \frac{P(D)}{4}\right.
$$

It follows from (2.5) that

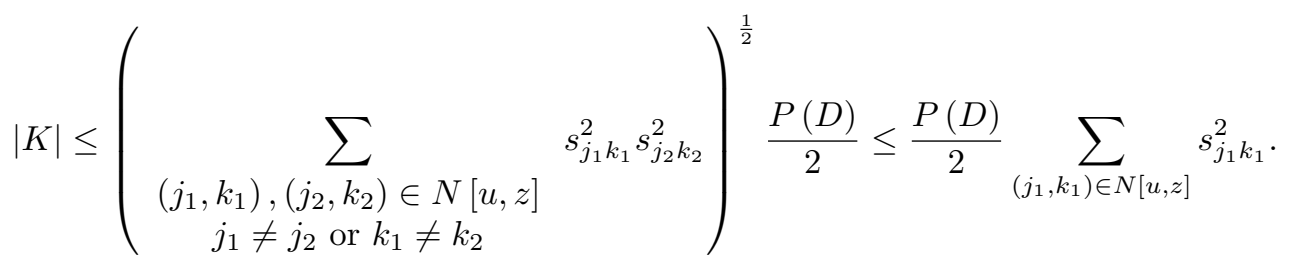

Combining this with (2.4) we get

$$
\begin{aligned}
\int_{D} T_{u, z, n, m}^{2}(x) d P(x) & =P(D) \sum_{(j, k) \in N[u, z]} s_{j k}^{2}+K \\
& \geq \frac{P(D)}{2} \sum_{(j, k) \in N[u, z]} s_{j k}^{2} .
\end{aligned}
$$


By (2.3) we have that

$$
\lim _{n, m \rightarrow \infty} \frac{1}{n^{2 q} m^{2 q}} \sum_{(j, k) \in N[u, z]} s_{j k}^{2}=0 \text { and } \lim _{n, m \rightarrow \infty} \frac{1}{n^{2 q} m^{2 q}} \sum_{j, k=1,1}^{n, m} s_{j k}^{2}=0 .
$$

Hence the result follows.

In the next examples we present a sequence so that it is $\left(C_{q}, 1,1\right)$ summable but almost none of its subsequences are $\left(C_{q}, 1,1\right)$ summable.

Example 2.7. Consider the double sequence $s_{j k}=(-1)^{j}(-1)^{k} \sqrt{j} \sqrt{k}$. Then

$$
\sum_{j=1}^{\infty} \frac{(-1)^{j} \sqrt{j}}{j^{q}}=\sum_{j=1}^{\infty} \frac{(-1)^{j}}{j^{q-\frac{1}{2}}} \text { is convergent in the ordinary sense for } q>\frac{1}{2},
$$

and

$$
\sum_{k=1}^{\infty} \frac{(-1)^{k} \sqrt{k}}{k^{q}}=\sum_{k=1}^{\infty} \frac{(-1)^{k}}{k^{q-\frac{1}{2}}} \text { is convergent in the ordinary sense for } q>\frac{1}{2} .
$$

On the other hand the double series $\sum_{j, k=1,1}^{\infty, \infty} \frac{(-1)^{j}(-1)^{k}}{j^{q-\frac{1}{2}} k^{q-\frac{1}{2}}}$ is convergent (see [1], page 90). Also since

$$
\sum_{j=1}^{\infty} \frac{(-1)^{j}(-1)^{k}}{j^{q-\frac{1}{2}} k^{q-\frac{1}{2}}} \text { is convergent in the ordinary sense for } k=1,2, \ldots
$$

and

$$
\sum_{k=1}^{\infty} \frac{(-1)^{j}(-1)^{k}}{j^{q-\frac{1}{2}} k^{q-\frac{1}{2}}} \text { is convergent in the ordinary sense for } j=1,2, \ldots
$$

then the double series $\sum_{j, k=1,1}^{\infty, \infty} \frac{(-1)^{j}(-1)^{k}}{j^{q-\frac{1}{2}} k^{q-\frac{1}{2}}}$ is convergent in the restricted sense by Theorem 1 of [5]. Since the double series $\sum_{j, k=1,1}^{\infty, \infty} \frac{(-1)^{j}(-1)^{k} \sqrt{j} \sqrt{k}}{j^{q} k^{q}}$ is convergent in the restricted sense, we get that the sequence $\left\{\frac{1}{n^{q} m^{q}} \sum_{j, k=1,1}^{n, m}(-1)^{j}(-1)^{k} \sqrt{j} \sqrt{k}\right\}$ converges to zero in the Pringsheim sense [6]. Hence the sequence $\left((-1)^{j}(-1)^{k} \sqrt{j} \sqrt{k}\right)$ is $\left(C_{q}, 1,1\right)$-summable to zero. On the other hand, for the case of $q=\frac{3}{4}$,

$$
\left(\frac{1}{n^{2 q} m^{2 q}} \sum_{j, k=1,1}^{n, m} j k\right)=\left(\frac{1}{n^{2 q} m^{2 q}} \frac{n(n+1)}{2} \frac{m(m+1)}{2}\right)
$$


the double sequence does not converge to zero. Hence we have, by Theorem 2.6, that

$$
\lim _{n, m} \frac{1}{n^{q} m^{q}} \sum_{j, k=1,1}^{n, m}(-1)^{j}(-1)^{k} \sqrt{j} \sqrt{k} r_{j k}(x) \neq 0 .
$$

So almost none of its subsequences are $\left(C_{q}, 1,1\right)$-summable to zero.

Example 2.8. Consider the double sequence $s_{j k}=(-1)^{j}(-1)^{k} j k$. Then

$$
\sum_{j=1}^{\infty} \frac{(-1)^{j} j}{j^{q}}=\sum_{j=1}^{\infty} \frac{(-1)^{j}}{j^{q-1}} \text { is convergent in the ordinary sense for } q>1,
$$

and

$$
\sum_{k=1}^{\infty} \frac{(-1)^{k} k}{k^{q}}=\sum_{k=1}^{\infty} \frac{(-1)^{k}}{k^{q-1}} \text { is convergent in the ordinary sense for } q>1 \text {. }
$$

On the other hand, the double series $\sum_{j, k=1,1}^{\infty, \infty} \frac{(-1)^{j}(-1)^{k}}{j^{q-1} k^{q-1}}$ is convergent (see [1], page 90). Also since

$$
\sum_{j=1}^{\infty} \frac{(-1)^{j}(-1)^{k}}{j^{q-1} k^{q-1}} \text { is convergent in the ordinary sense for } k=1,2, \ldots
$$

and

$$
\sum_{k=1}^{\infty} \frac{(-1)^{j}(-1)^{k}}{j^{q-1} k^{q-1}} \text { is convergent in the ordinary sense for } j=1,2, \ldots
$$

then the double series $\sum_{j, k=1,1}^{\infty, \infty} \frac{(-1)^{j}(-1)^{k}}{j^{q-1} k^{q-1}}$ is convergent in the restricted sense by Theorem 1 of [5]. Since the series $\sum_{j, k=1,1}^{\infty, \infty} \frac{(-1)^{j}(-1)^{k} j k}{j^{q} k^{q}}$ is convergent in the restricted sense, we get that the sequence $\left\{\frac{1}{n^{q} m^{q}} \sum_{j, k=1,1}^{n, m}(-1)^{j}(-1)^{k} j k\right\}$ converges to 0 in the Pringsheim sense [6]. Hence the sequence $\left((-1)^{j}(-1)^{k} j k\right)$ is $\left(C_{q}, 1,1\right)$-summable to 0 . On the other hand, for the case of $q=\frac{3}{2}$,

$$
\left(\frac{1}{n^{2 q} m^{2 q}} \sum_{j, k=1,1}^{n, m} j^{2} k^{2}\right)=\left(\frac{1}{n^{2 q} m^{2 q}} \frac{n(n+1)(2 n+1)}{6} \frac{m(m+1)(2 m+1)}{6}\right)
$$

the double sequence does not converge to zero. Therefore, Theorem 2.6 implies almost none of its subsequences are $\left(C_{q}, 1,1\right)$-summable to zero. 


\section{References}

[1] Bromwich, M.A., An introduction to the theory of infinite series, Macmillan Co., 1942.

[2] Buck, R.C., Pollard, H., Convergence and summability properties of subsequences, Bull. Amer. Math. Soc., 49(1943), 924-931.

[3] Crnjac, M., C̆unjalo, F., Miller, H.I., Subsequence characterizations of statistical convergence of double sequences, Radovi Math., 12(2004), 163-175.

[4] Garling, D.J.H., Inequalities: A Journey into Linear Analysis, Cambridge University Press, 2007.

[5] Móricz, F., On the convergence in a restricted sense of multiple series, Analysis Mathematica, 5(1979), 135-147.

[6] Móricz, F., The kronecker lemmas for multiple series and some applications, Acta Math. Acad. Sci. Hungar., 37(1981), 39-50.

[7] Orhan, C., Tas, E., Yurdakadim, T., The Buck-Pollard property for p-Cesàro matrices, Numer. Funct. Anal. Optim., 33(2012), 1-7.

[8] Patterson, R.F., A characterization for the limit points of double sequences, 32(1999), 775-780.

[9] Pringsheim, A., On the theory of double infinite sequences of numbers. (Zur theorie der zweifach unendlichen zahlenfolgen.), Math. Ann., 53(1900), 289-321.

[10] Tas, E., Orhan, C., The Buck-Pollard property for $(C, 1,1)$ method, submitted.

[11] Tsuchikura, T., Arithmetic means of subsequences, Tôhoku Mathematical Journal, 2(1950), 188-191.

[12] Unver, M., Inclusion results for four dimensional Cesàro submethods, Stud. Univ. BabeşBolyai Math., 58(2013), 43-54.

[13] Zhizhiashvili, L.V., Some problems in the theory of simple and multiple trigonometric and orthogonal series, Russ. Math. Surv., 28(1973), 65-127.

Emre Taş

Ahi Evran University, Department of Mathematics

Bağbaşı, 40100 Kırşehir, Turkey

e-mail: emretas86@hotmail.com

Cihan Orhan

Ankara University, Faculty of Science, Department of Mathematics

Tandoğan, 06100 Ankara, Turkey

e-mail: orhan@science.ankara.edu.tr 